\title{
On the Development of the Advanced Encryption Standard
}

\author{
YIQUN LISA YIN
}

NTT Multimedia Communications Laboratories

250 Cambridge Avenue, Palo Alto, CA 94306, USA

yiqun@nttmcl.com

The Advanced Encryption Standard (AES) will be one of the most important cryptographic algorithms for the next few decades. As the successor to the aging Data Encryption Standard (DES), the AES will be integrated into both government systems and various commercial products to protect the privacy of electronic information and communication.

The development of the AES has been organized by the National Institute of Standards and Technology (NIST) of the United States. In 1997, NIST provided detailed security and efficiency requirements for the AES and requested proposals from the public. In 1998, 15 proposals submitted from many countries were accepted as candidate algorithms. After a year of initial evaluation, five of the submissions were selected by NIST as finalists. We will take a closer look at the candidate algorithms, especially the five finalists - MARS, RC6, Rijndael, Serpent and Twofish. By analyzing novel features of these ciphers, we will be able to see the trend in the design of modern encryption algorithms.

It is worth noticing that the public cryptographic community has been actively involved in the development of the AES. This is quite a contrast to the development of DES back in the 1970s. In particular, a variety of cryptographic techniques in the open literature have been used to analyze the security of the AES candidates. Such techniques include differential cryptanalysis, linear cryptanalysis, related-key attacks, power analysis, and

The original version of this chapter was revised: The copyright line was incorrect. This has been corrected. The Erratum to this chapter is available at DOI: 10.1007/978-0-387-35515-3_53 
many others. We will give a brief survey of these newly developed techniques and discuss their significant impact on cipher design and analysis.

The AES effort is perhaps one of the most important competitions in the history of cryptography. From an academic viewpoint, it has played a unique role in stimulating cryptographic research. From an industrial viewpoint, it will play a key role in securing future electronic commerce. Many factors will affect the final decision that will be made by NIST in summer 2000 . We look forward to the winner of the AES. 